\title{
Landscape, Interaction, Complexity
}

\author{
The discipline emerges as a restless body of observations upon particular \\ classes of data, between a certain range of scales, held together by a network \\ of changing methodology and implicit theory. \\ -DAVid ClARKe, "ARCHAEOlogy: the loss of inNocenCE"
}

This chapter outlines the theoretical framework of the present work: an integrative approach that incorporates archaeologies of landscape, interaction, and complexity. From a landscape perspective, I examine the distribution of settlement evidence in central Greece and how this evidence changes through the Late Bronze Age and Early Iron Age. As an archaeology of interaction, this book focuses on the modeling and articulation of networks on multiple scales-local, regional, and long-distance-and how these are manifest in the archaeological record. These approaches to landscape and interaction combine in what is ultimately an archaeology of social complexity, focused on the constitution and development of societies in the late second and early first millennia BCE.

\section{AN ARCHAEOLOGY OF LANDSCAPE: \\ COMMUNITIES IN CONTEXT}

As an archaeology of landscape, the following chapters analyze archaeological evidence for settlement, burial, politics, and production, as distributed through the regions of central Greece. This study of settlement patterns involves the quantification and comparison of settlement evidence, alongside spatial models of territory and connectivity. More qualitative aspects of landscape archaeology are engaged through the themes of space, place, and landscape-categories that are socially constructed in ways informed by the physical environment, human experiences, and collective memory. While physical spaces form the contexts in which humans live, operate, and interact, place-making activities condense interactions in the landscape by bringing together individuals participating in local and regional networks of particular practices, such as communal feasting or festivals at regional 
sanctuaries. Iterative movements through the landscape traverse local, regional, and more distant spaces, habituating connections between individuals and groups. Like other types of interactions, practices of this sort are related to social complexity in the sense that they are both inclusive and exclusive, serving to create and reinforce social bonds, inequalities, and associations with place. In a similar way, technology and production deserve special attention. Agricultural activity and craft production bring together people and things across local and regional landscapes, as raw materials, products, and technological knowledge travel. Mobility and movement between places, spaces, and landscapes are the means by which social life is constituted and are necessarily multiscalar. In this section I focus first on the fundamental relationship between geography and society and then move on to define my approach to the region as the crucial scale of analysis. I discuss the regional datasets and methods of analysis in detail in chapter 2.

\section{Connective Contexts in Mediterranean Geography}

Maritime and terrestrial geography provide complementary sets of landscapes, coastscapes, and seascapes in which the movements and dynamics of human societies play out. On the largest scale, we are concerned with the Mediterranean writ large, the fractal geography of which has resulted in a particular form of connectivity (map 1) (Braudel 1972; Horden and Purcell 2000; Broodbank 2013). Microecologies of certain landscapes - coastal plains, mountain plateaus, river valleys, upland steppes-characterize a highly fragmented geographical area, where different zones are better suited to different modes of production. At the same time, these areas tend to be geographically distinct from one another, often with clear natural boundaries that easily allow the demarcation of territory or regional identity. Nevertheless, this fragmentation drives interaction between microregions, in order to diversify patterns of consumption (of agricultural and craft products) and socialization (through marriage relationships and alliances). As a response, communities develop practices of social storage and risk buffering that ensure that bad years or crop failure in certain regions can be ameliorated by exchange relationships with others (Halstead 1989; Broodbank 2000, 84). In practical terms, such interaction is most likely to be carried out along particular paths, again owing to the particular topographic fragmentation of Mediterranean microregions.

The Aegean Sea and the particularly varied geographies of mainland Greece are at a crossroads whose significance is paramount in the long-term history of the Mediterranean. As a bridge between Asia and Europe, the Aegean and the Greek mainland have been traversed time and again by migrants coming across both land and sea-from the earliest human populations of the area (crossing through northern Greece) to the first farmers in Europe (in Crete and in Thessaly) to present-day refugees fleeing various conflicts. When the eastern Mediterranean world expanded into one of states, empires, and traders in the Late Bronze Age and Early Iron Age, the Aegean's centrality was particularly significant, forming a bridge between the eastern and central Mediterranean. 
As our focus narrows to the study region at the center of this book, so, too, can the demonstrable geographies of centrality and connectedness. From a maritime perspective, the Euboean Gulf is a conduit that runs between the central Greek mainland and the island of Euboea (map 1); it is a sheltered channel that also links the northern and southern Aegean Sea. This corridor is surrounded on all sides by a diverse set of landscapes that make up the regions of Attica, Boeotia, Malis, Thessaly, and Euboea (see further in chapter 2). It has been a critical nexus of human interaction in many phases of history, where geography, individuals, groups, and materials have come together in the course of day-to-day local practices, as well as in long-distance voyages that spanned the Aegean and in some cases the entire Mediterranean. ${ }^{1}$ From a terrestrial perspective, the landscapes of central Greece are home to a diverse array of agricultural land, mountain pastures, and routes of movement. Parallel to the Euboean Gulf, the Great Isthmus Corridor (between, roughly, Delphi and Lamia) brings the region of Phokis and Mount Parnassos into the fold and provides the same type of northwest-southeast land route as the Euboean Gulf does on the eastern side of the study area.

\section{A Regional Perspective: Multiple Scales, Hierarchies of Space}

On a fundamental level, this book is a contribution to the archaeology of settlement in central Greece in the Late Bronze Age and Early Iron Age. From there it branches out to wider sets of relations. I use evidence from archaeological surveys and excavations to examine how landscapes were inhabited and traversed and how they changed over time. The regional perspective put forward here is multiscalar in that it seeks to integrate archaeological and landscape evidence across a variety of social and spatial scales that often overlap (figure 1). Regions themselves can be defined on multiple scales, which are nevertheless geographically coherent. Their boundaries - distinguishable, but permeable - can be identified by features in the landscape (mountains, streams, or the sea), and in size they range from microregions, such as a mountain plain or a river valley, to mesoscales comprised of several microregions, like Boeotia or Thessaly, to macroregions, like central Greece. These classifications exist alongside conceptions of social space, which come together in the context of landscapes.

In using the term "landscape," I refer mainly to the combination of the physical environment and lived activities and experiences within it. The archaeology of landscape presented here examines the distribution of evidence for these activities and experiences diachronically and regionally, as well as with the goal of understanding human experiences in their broader cultural and environmental contexts. In practical terms, this begins with mapping the distribution of

1. On sea routes in the Aegean and maritime connectivity, see Agouridis 1997; Arnaud 2005; Papageorgiou 2008; Sauvage 2012; Tartaron 2013 (especially on local connectivity and the particular importance of short-distance seafaring). On the physical geography of Greece and its relationship to human history, see Philippson 1951; Bintliff 1977b; Horden and Purcell 2000; Knodell 2017. 


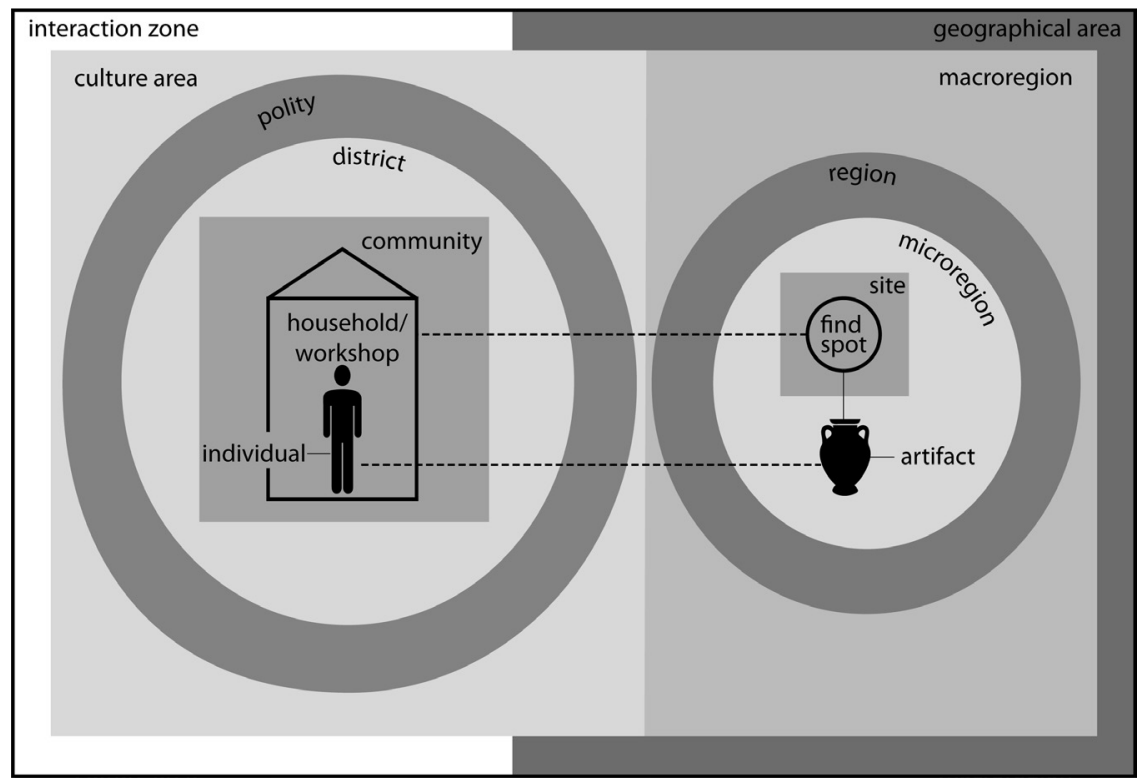

FIGURE 1. Nested (but often overlapping) scales of social and spatial units: spatial modes of organizing territory on the right, with socially defined entities on the left; these categories naturally overlap and coexist: e.g., multiple artifacts within one of several findspots at a site may provide information about individuals or a household within a community; a region may contain multiple polities, each comprised of a number of communities, which in some cases may be grouped into administrative districts (illustration by Denitsa Nenova).

archaeological remains, then analyzing and interpreting them in terms of their relationships to other places, both near and far (see further in chapter 2).

The archaeology of regions has been a major focus of research for some time in Greek archaeology, though issues of scale remain a challenge (Cherry 1983b; Bennet and Galaty 1997; Alcock and Cherry 2004; Tartaron 2008; Knodell and Leppard 2018). Large-scale regional studies-for example, of mainland Greece or the island of Crete-need to integrate a wide variety of datasets from surveys and excavations, usually to address particular historical problems. The most successful examples have examined topics such as different pathways to complexity in the north and south Aegean, the emergence of the polis, breakages in the Hellenistic world, and the landscapes of Roman Greece (Alcock 1993, 1994, 2002; Halstead 1994; Bintliff 1997). Further successful examples integrate data across smaller regionsfor example, the northeast Peloponnese (Cherry and Davis 2001; Wright 2004b). It remains difficult, however, to integrate diverse datasets across large geographical areas with more than broad strokes between uncharacterized dots on a map.

Archaeologists have increasingly recognized the need to integrate regional data across multiple scales (e.g., Parkinson 2018). The vast majority of human activity occurs within local or regional extents. While many face-to-face interactions 
take place within the bounds of a household or a neighborhood, the spatial extent of daily life ranges far beyond the individual site or community, especially in agropastoral societies. Household production is highly localized at the level of an individual site, but it requires raw materials from farther afield. Agricultural production extends beyond dwelling locations and may involve cultivation in areas quite distant from a habitation location. Pastoral activities are by nature distributed across the landscape in the constant quest for fodder. At a broader scale, individuals move between communities in order to arrange suitable marriage partners and to ensure a diversification of products and subsistence strategies. Findspots or sites (both archaeological categories) may or may not represent an individual community (a social category) (see figure 1). At the same time, individuals move across and between regions as well, which can be conceived of in social terms as polities, culture areas, or interaction zones.

Interaction between different types of social groups requires us to consider notions of community identity, polity, and territory, all of which are embedded deeply in notions of place, space, and landscape. Studying how these spheres of spatial production and interaction are interwoven is a central feature of the landscape archaeology proposed in this book. An equally important focus concerns how the interconnectivity between places leads to the redefinition of regions in territorial and political terms (rather than, say, physical ones) (see, e.g., de Montmollin 1989; Smith 2003; Bevan 2010; Kosiba and Bauer 2013). The settlement evidence is therefore presented qualitatively, quantitatively, and relationally in the chapters that follow. Site types and hierarchies are defined for each period, then evaluated in terms of what type of activities and communities they most likely represent. Relationships between communities are modeled as well, in order to explore the interactive workings of early Greek societies.

\section{AN ARCHAEOLOGY OF INTERACTION: NETWORKS AND TECHNOLOGIES}

As an archaeology of interaction this book examines how sites, communities, and landscapes are woven together on multiple scales. On the one hand, this is integrated into the settlement pattern analysis. On the other, I look to two other proxies for interaction for each of the periods in question. First, technology and media offer evidence of and catalysts for mobility and knowledge-sharing. Second, more traditional forms of evidence for long-distance interaction-imports, exports, diplomatic texts, and historical or literary accounts-are also examined where available. In each case, I am concerned first with connectivity within and between the regions and microregions of central Greece and second with the place of central Greece in the wider Aegean and Mediterranean world.

In chapters 3, 4, 5 and 6 I model networks of interaction in geographical space in a Geographic Information Systems (GIS) framework. Models of local 
and regional interaction networks illustrate how and where people likely moved through and between the landscapes and communities of central Greece. I examine longer-distance interactions through an interpretative analysis of material culture, especially in relation to production and media technologies (involving questions of know-how, style, and regional identity). Aspects of network theory concerning centralization, small worlds, and the strength of weak ties are invoked at various points to explain the models and evidence presented here in relation to the Greek landscape and the societies inhabiting it.

This approach differs from many other studies of interaction in embracing both qualitative and quantitative approaches. Such a strategy is appropriate in considering the potpourri of interaction studies in archaeology and anthropology. ${ }^{2}$ While much excellent work has been done, there remains no unified body of theory on the topic. This is probably because interaction could be used to describe nearly all aspects of human behavior-from face-to-face conversations (Goffman 1967) to long-distance trade (Renfrew 1975). An explicitly integrative, multiscalar approach is a productive way forward.

\section{Multiscalar Networks and Diachronic Change}

Over the last decades, networks have emerged as a powerful approach to the study of interaction, especially in archaeological contexts (Peeples 2019; Donnellan 2020). At their most fundamental level, networks are a way of abstracting entities (nodes) and relationships between them (links). Here, the graphic representation and analysis of these relationships form the basis of the network study. As with the term "interaction," however, "networks" can refer to a wide range of theoretical or methodological approaches (see, e.g., Knappett 2011, 37-58; Knodell 2013, 65-96; 2017; Brughmans 2013; Brughmans, Collar, and Coward. 2016; Mills 2017). Most archaeological and historical applications of networks fall into three general categories:

1. The term "network" is used metaphorically to describe interconnectivity among individuals or groups: the Late Bronze Age eastern Mediterranean was home to a complex network of interactions between various cultures and polities (e.g., Horden and Purcell 2000; Broodbank 2013).

2. Concepts from social network theory (especially centrality, small worlds, scalefree growth) are used to describe social relationships, primarily as an interpretative

2. Approaches include description-based catalogs of imports, diffusionist models (Childe 1925, 1936, 1958), interaction spheres (Caldwell 1964), trade studies (Renfrew 1969, 1975; Chang 1975), systems perspectives (Clarke 1968, 1972; Flannery 1968; Renfrew 1972), peer-polity interaction (Renfrew and Cherry 1986), world-systems theory (Wallerstein 1974; Kohl 1987; Blanton and Feinman 1984; Sherratt and Sherratt 1993; Kristiansen and Larsson 2005; Kardulias and Hall 2008; Parkinson and Galaty 2010), its critiques (Schneider 1977; Stein 2002), globalization (Hodos 2017, 2020), and various types of network theory (Knappett 2011, 2013; Blake 2014; Brughmans, Collar, and Coward 2016; Iacono 2019; Peeples 2019). On the persistent problem of the diffuse nature of the concept, see Schortman and Urban 1987, 1992; Schortman 1989; Iacono 2019, 8-13). 
aid: Mycenaean palaces reorganized regional networks to make them highly centralized, putting a large amount of power (and vulnerability) in the central node (the palace) (e.g., Malkin 2011; Tartaron 2013; Kramer-Hajos 2016).

3. Formal or quantitative methods of network analysis are deployed in the generation and examination of models based on material evidence: analysis can show patterns of clustering across the study area, variable levels of centrality in different nodes in the network, or the convergence of different attributes of material culture (e.g., Broodbank 2000; Knappett 2011; Blake 2014; Brughmans, Collar, and Coward 2016; Iacono 2019).

This final category ranges widely, often incorporating approaches from social network analysis (SNA) or complexity studies and network science. The main uses of networks in this book are as a dual geographical and relational approach that combines elements of all three categories, though chiefly 2 and 3 .

For the most part, the nodes discussed in this book are archaeological sites-in particular, the ones in the dataset that represent past communities. At times, nodes can refer more specifically to institutions or individuals or more broadly to regions in the wider Mediterranean context. This flexibility is necessary in attempting to reconstruct networks on multiple scales and of different types. Links must be defined with equal flexibility. The individuals, groups, things, and places that act as nodes are mediated by a variety of other individuals, groups, things, and places, which necessarily result in multiple types of interactions. The types of links represented here can be strictly material (e.g., a direct contact situation of trade or raw material procurement) or more ephemeral (shared technologies or aspects of material or visual culture).

Networks are used here to articulate multiple geographical scales, while maintaining a relatively unified theoretical framework. ${ }^{3}$ In general, discussions in the following chapters refer to (1) local networks, which link individuals within or between nearby communities; (2) regional networks, which join communities, landscapes, and polities; and (3) interregional networks, which connect polities or cultural groups across the Aegean or even the Mediterranean. These should not be seen as stark divisions but rather as groupings with relatively fluid boundaries (see figure 1). It is the mesoscale-which is best understood through a study of the archaeological landscape-that bridges local and regional distances and that receives the most explicit treatment here in terms of modeling (see further in chapter 2).

3. Knappett (2011) has taken a different approach to networks on multiple scales (largely centered around ceramic production and distribution in the Minoan Aegean), analyzing microscale networks of proximate interactions, mesoscale networks concerned with communities of practice, and macroscale networks of regional interactions. More recently, Donnellan (2016) has examined funerary practices at the multicultural settlement of Pithekoussai from the perspective of multiscalar networks. 
Certain general trends in network behavior can be drawn on as interpretative tools or principles. Four patterns feature here: scale-free growth, centrality, small worlds, and the strength of weak ties (see also Malkin 2011, 3-28; Knodell 2013, 346-47; Kramer-Hajos 2016, 20-21). Scale-free networks essentially describe "the rich get richer" phenomenon, whereby places that are well connected are likely to become better connected, often at an exponential rate (Barabási and Albert 1999). In terms of centrality, highly centralized networks have few hubs through which all interactions must go (Baran 1964). This puts much control over interaction in a single node, which can thus be very powerful and efficient. However, the entire system is left vulnerable to collapse since, if the hub is removed, the whole system breaks down. Decentralized networks, in which hubs emerge almost naturally in many different places, are much more common. Distributed networks have fewer or no hubs.

A small world is a type of network in which most nodes are connected to their nearest neighbors but certain links exist that connect other small worlds to each other (Milgrim 1967; Pool and Kochen 1978; Watts and Strogatz 1998). A concept that operates within small-world networks is the strength of weak ties (Granovetter 1973). While interactions between near neighbors are likely to be more frequent (or stronger), the less frequent, often longer-distance interactions between other small-world networks - that is, the weak ties_can be particularly significant. Such weak ties can introduce new ideas or materials to a small-world network.

While network theory is increasingly prevalent in archaeology and ancient history, the integration of geographical realities into such an approach is significantly rarer (but see Bevan 2010; Mills et al. 2015; Peeples 2019, 471-80; Brughmans and Peeples 2020). Moreover, most archaeological studies of networks seek to document or analyze interaction as recorded in things - for example, through shared elements of ceramic style or technology (Knappett 2011; Blake 2014). My goal is rather to model assumed interactions in space heuristically, based on the idea that communities would have interacted with neighboring communities. In particular, I use (1) nearest-neighbor networks based on habitual contact with a certain number of proximate sites and (2) a connectivity model of routes in the landscape (on methods, see further in chapter 2).

\section{Texts, Technologies, and Things}

Beyond network models, I examine several other proxies for interaction, which are chiefly regional and interregional in scope. These comprise words, images, and things. Texts that refer to interaction and population movement signal an eastern Mediterranean in transition, with mobility and migration as powerful forces (Demand 2011; Aslaksen 2016; Isayev 2017; Driessen 2018; Wallace 2018). At the same time, technologies - and their dispersal through different regions and culture areas-are important proxies for interactions (Feldman 2007, 2014; Brysbaert 2011; Ferrara 2015). One final relevant element of interaction studies highlights 
things as social actors at the core of the archaeological project (Witmore 2007; Hodder 2012; Olsen et al. 2012). Things are both media of interaction, through which messages move and meanings are transferred, and they themselves play important roles in social interactions, expressing meanings that can range from indeterminate exoticism to projecting particular political messages (A. Smith 2015; Khatchadourian 2016).

Images and texts have special significance, since they carry specific messages by design. In some cases, the meanings these media carried were deliberate and clear; in others, the meanings were unintentional and subtle. Changes in media and technology as mobile and lasting carriers of cultural information are therefore paramount to tracking and explaining social change alongside landscapes of settlement and models of interaction.

\section{AN ARCHAEOLOGY OF COMPLEXITY: SOCIETIES IN TRANSITION}

As an archaeology of complexity, this book examines the development of societal organization from the Late Bronze Age to the Early Iron Age. Archaeologies of landscape and interaction serve as the context and articulation of what is ultimately an archaeology of complex societies-that is, an approach to ancient communities that aims to understand their sociopolitical organization, variation, and change over time.

Complex societies have historically been viewed in contrast to simple societies, though these terms can each apply to a range of sociopolitical formations. Complex societies are generally defined as human groups in which levels of social hierarchy are present, along with a variety of roles and specializations represented among the individuals that comprise them. Social complexity has comparative value as a flexible concept applicable to a wide variety of cultural circumstances (see, e.g., Trigger 2003; A. Smith 2003; M. E. Smith 2011; Small 2019). Because of its relationship to variation and hierarchy, complexity is often a stand-in for levels of social inequality (Chapman 1990, 2003). This complexity usually refers to states and other social formations with clearly demarcated differentiation in social status and roles, though it can (and should) apply also to a range of modes of sociopolitical organization, both loosely and strictly defined.

Complex adaptive systems offer an inroad to the study of ancient societies where an understanding of the individual parts does not necessarily result in the understanding of the whole (Kauffman 1993; Martin and Sunley 2007; Mitchell 2009). Shifting networks of interactions result in change across the system in its entirety, which is comprised of various types of agents. In considering the system as a dynamic whole, patterns such as emergent behaviors, phase transitions, and collapse can be identified, a fact that has obvious implications for understanding complex societies as dynamic systems (Bentley and Maschner 2003; Kohring 
and Wynne-Jones 2007; Kohler 2012). Complexity thinking thus provides a useful framework within which to describe and understand the dynamic behaviors of past social groups.

The archaeology of social complexity examines how interconnectedness relates to the emergence of new properties in a social system (van der Leeuw 1981; Cherry 1983a, 1986; Chapman 1990, 2003; Halstead 1994; Bintliff 1997; Smith 2003; Ames 2008; Knodell and Leppard 2018). Archaeologists of complex societies are particularly concerned with how societies become comprised of larger, more internally diverse, and more complex (yet integrated) social structures, and how social inequality is present through the manipulation of these structures. Such processes are nonlinear and multidirectional. That is to say, simplification is as worthy an object of study as growth. Renfrew (1979) made a similar point concerning the collapse of ancient states and the aftermath, pointing particularly to patterns of "catastrophe and anastrophe." Similarly, in modeling the rise of the polis in early Greece, Snodgrass (1980a, 44-47) has emphasized the need to pay attention also to communities that did not become poleis, a call picked up more thoroughly by Morgan (2003). In general, then, complexity should be viewed on a nonlinear, variable scale. Complexity does not conform to strict definitions or sets of attributes but has to do with structure, interaction, and power-scales of settlement and territory, the integration of institutions, and levels of inequality.

Like other complex systems, social complexity is fluid and dynamic, and it develops in an ongoing way. Reorganization is therefore a major theme; scale also factors in the relationship between time and change (Lock and Molyneaux 2006). If we consider Braudel's (1972, 20-21) tripartite conception of timescales-the longue durée, conjoncture, and histoire événementielle (long-term, societal, and individual rhythms of time) - we might draw parallels between these and their social-historical correlates. In the long term, societies continuously reconstitute themselves-quite slowly, as their institutions become entrenched and reformulated through daily practices (Bourdieu 1977; Giddens 1984; Nakassis 2013b, 246). These processes of entrenchment and reconstitution occur on the resilient and rhythmic scales of the long and medium term. Such continuous processes are often not visible in the archaeological record and, when they are visible, they read as slow-moving, gradual development (e.g., long-lasting practices concerning agropastoral lifeways or household organization). More rapid, large-scale change is often observable archaeologically; it can be compared to patterns of punctuated equilibrium in evolutionary theory (Cherry 1983a; Gould 2007; Manning 2018). Such punctuated equilibria can be detected in the medium term or the rhythmic/ iterative timescale that we would associate with more distinct and recognizable social change (e.g., in political organization or material culture traditions). Finally, an archaeological perspective rooted in complexity must recognize the capacity for short-term, agentive, and events-based change to have dramatic impacts across 
a much wider system (e.g., political transitions, conflicts, and disasters). While specific events are rarely detectable archaeologically, their consequences often are, especially when they are viewed in combination with hints afforded by other sources of information, such as contemporary and later written records.

\section{Social Complexity in Early Greece}

Despite a long tradition of archaeological research, our understanding of early Greek sociopolitical organization is rather poor. We know more about some periods and places than others, and there is a wide range of evidence and of organizational scales represented across the Aegean world. In general, discussions of political organization tend to focus on palatial administration and kingship in the Mycenaean Palatial period, more localized forms of leadership in the Postpalatial period and Early Iron Age, and the development of the polis beginning in the eighth century (in which various forms of tyranny, oligarchy, aristocracy, and democracy would eventually appear). Many of these discussions, however, involve the projection or retrojection of political forms known from earlier or later textual sources across the early Greek world as a whole (wanax, basileus, etc.). In taking into account the diversity of archaeological evidence, I argue that regionalism should be the priority over any uniform view of early Greek society.

Most discussions of Mycenaean palatial systems focus overwhelmingly on (and extrapolate from) what we know about certain archetypes-Mycenae from the perspective of material culture and Pylos from the perspective of Linear $\mathrm{B}$ texts that tell us about the operation of the palace. These were relatively centralized polities with an administrative system whose activities were documented via the nonpermanent medium of unfired clay tablets (Ventris and Chadwick [1956] 1973; Shelmerdine 2008; Parkinson and Galaty 2007; Nakassis 2013a; Killen 2015). Social hierarchies can be distinguished in the archaeological record by elaborately constructed burials with wealthy grave goods, and family tombs suggest some degree of inherited status. The monumental fortifications of the period and other large-scale building projects suggest the ability to organize and control large labor forces for complex tasks. This general picture appears to be the case in most areas where we have Mycenaean palaces. However, we have little reason to believe that all Mycenaean palaces functioned in the exact same way and many reasons to believe they functioned quite differently from each other (see further in chapter 3 ).

Beyond the operation of the palaces themselves, secondary state formation is a significant topic of broader anthropological interest, to which the Mycenaean case can contribute (Parkinson and Galaty 2007). Explicit studies of Mycenaean state formation as such, however, are relatively rare, usually being limited to particular site-based or regional case studies, mostly in the Peloponnese (Laffineur and Niemeier 1995; Cherry and Davis 2001; Cosmopoulos 2005, 2006; Galaty and Parkinson 2007; Fitzsimons 2011; Pullen 2011b; Ruppenstein 2012; Kramer-Hajos 2016). 
For the Postpalatial Bronze Age, debates revolve largely around notions of collapse and recovery, with a "revival" period somewhere in between. Other than among specialists, this period is often rendered indistinct from either the Palatial period (because it is still Mycenaean) or the Early Iron Age (because it is still part of the postcollapse "Dark Age"). Specialists who do make these distinctions have lamented that the reorganization that followed the end of the Palatial period has been a "lost opportunity" in studies of urbanism in the Aegean (Lemos et al. 2009). ${ }^{4}$ Kramer-Hajos (2016) has recently pointed out that the sociopolitical organization of this period in fact looks quite similar to what immediately preceded the emergence of the palatial systems, at least in central Greece-complex communities in which local elites engaged in regional and interregional competition and trade. It seems to me that this world, much more than the Palatial age, is reflected in Homer's imagination of a heroic past-a time of violent conflict, loose political organization, and high levels of mobility (see also Crielaard 2006, 2011b).

A range of sociopolitical complexity also seems to have been present in the Early (Prehistoric) Iron Age. Well-connected centers with demonstrable social hierarchies, such Lefkandi, Athens, Argos, and Knossos, represent one end of the spectrum, while the vast majority of other sites seem to have operated on a much smaller scale. ${ }^{5}$ These hierarchies are manifest more on local scales of the individual site rather than across entire regions. For example, politically coherent settlement hierarchies seem to be nonexistent. While a site hierarchy is employed in this book in order to differentiate between major settlements, minor sites, and isolated findspots representing a range of activities, there is less that we can say explicitly about, say, a single main site and subsidiaries within a particular locale. Previous treatments of sociopolitical organization tend to focus on hierarchy as manifest within an individual settlement and look also to later textual references to the basileus (as described by Homer and Hesiod) as a sort of "big man" or chief-like figure (Mazarakis Ainian 1997; Dickinson 2006; Crielaard 2011b; Kõiv 2016).

The picture changes considerably in the eighth century (Protohistoric Iron Age), when Greece began the transition from a world of villages to a world of towns. By 700 BCE there were probably dozens of towns with populations of over 1000 individuals, whereas in the previous period there were likely only three or four: Argos, Knossos, Athens, and Lefkandi (Morris and Knodell 2015, 348-51;

4. On urbanism in Aegean prehistory, see Branigan 2001; Owen and Preston 2009; Letesson and Knappett 2017. For comparative views, see Marcus and Sabloff 2008; Yoffee 2015. For a recent critique of urbanism in certain (Middle Bronze Age) contexts in the Aegean, see Cherry 2017.

5. The exemplary sites are also the best published. On Lefkandi, see Popham, Sackett, and Themelis 1980; Catling and Lemos 1990; Popham, Calligas, and Sackett 1993. On Athens, see Papadopoulos 2003; Papadopoulos and Smithson 2017. Nichoria is a well-published example of a much smaller-scale settlement: see McDonald, Coulson, and Rosser 1983. For a recent summary of archaeological and later textual evidence for political organization in the Early Iron Age, especially the question of leadership and basileia, or Homeric kingship, see Kõiv 2016. 
see also Whitley 1991, 184-90; Mazarakis Ainian 1997). Such growth in the number and population of communities in central Greece necessitated restructuring political and settlement strategies, and it is at this point that we see the nascent development of (in some areas) early Greek poleis and (in others) regionally collective ethne, groups that exhibited a variety of modes of social organization (Morgan 2001, 2006; Sakellariou 2009; Papadopoulos 2016a). While standard narratives of the early polis lead us to believe that the eighth century was a sort of crucible for political development, other sources, such as the poetry of Hesiod and Homer, suggest that we are still dealing mostly with small-scale village societies, although we do see the emergence of clearer gradations in social class and wealth within some larger communities, such as Athens or Eretria. At the same time, an interregional political consciousness became more pronounced, as can be witnessed in the growth of Panhellenic and regional sanctuaries. It was not until the subsequent Archaic period, however-beginning in the seventh century rather than in the eighth - that we really see the growth of the kinds of communities and political institutions (tyranny, oligarchy, aristocracy, democracy) that have come to characterize our understandings of ancient Greek city-states (Snodgrass 1980a; Hansen and Nielsen 2004).

\section{Early Greece in the Archaeology of Complex Societies}

Greece plays a rather minor role in the comparative archaeology of complex societies. It makes occasional appearances in the archaeology of early cities and the archaeology of empire, along with approaches to interpolity relationships and state formation, but it is rarely at the forefront (though see, e.g., Renfrew and Cherry 1986; Hansen 2000; Alcock et al. 2001). Part of the reason for this is that early Greek political formations do not compare well with the "Archaic States" that are often the subject of comparative literature in anthropological archaeology (e.g., Trigger 2003; Feinman and Marcus 1998; Yoffee 2005; M. Smith 2011; Routledge 2014). Cross-cultural, anthropological discussions of state formation tend to focus on the "pristine" states of Mesopotamia, Egypt, the Indus Valley, China, Mesoamerica, and the Andes. These are large states with extensive territories that have very little in common with the polities of early Greece. Secondary state formation and the operation of nonstate complex societies are discussed much less frequently. Moreover, for all the attention that is given to state formation and collapse, there is remarkably little theoretical attention given to oscillations in sociopolitical complexity and to the question of how societies reconstitute themselves after periods of collapse or decline. ${ }^{6}$ These themes are precisely where the Aegean is well poised to contribute to wider, comparative discussions.

6. There are, however, occasional examples of dynamic models, dual processual models, and evolutionary approaches in Aegean archaeology: see, e.g., Parkinson and Galaty 2007; Small 2009, 2019, drawing on Marcus 1998; Blanton et al. 1996. 
This book provides a case study in the archaeology of political formations that are smaller in scale and complexity than (1) state-level societies that feature in most of the comparative literature and (2) many of the contemporary states with which Aegean societies were in contact. Indeed, it is worth questioning whether the term "state" is appropriate for any period under study, at least as compared to the other eastern Mediterranean "states" with which the Aegean world was in contact. But if these are not good analogues, what are?

Similar scales of societies and political trajectories (secondary state formation, collapse, decline, reconstitution) can be found in several other contexts across the globe, although in a variety of guises. The archaeology of "nonstate" social complexity can encompass everything from hunter-gatherers to highly complex chiefdoms (Price 1981; Earle 1997; Kirch 2010). The problems of the bandtribe-chiefdom-state models of social evolution have long been acknowledged, and I do not advocate for any kind of neoevolutionary conceptual framework here (Sahlins and Service 1960; Sahlins 1972; Fried 1975). Nevertheless, some of these terms can and do retain descriptive and explanatory utility when not viewed strictly in evolutionary terms (Earle 1987; Parkinson 2002; Fowles 2002). Other researchers have sought less loaded terminology. Ranked societies; middle-range, intermediate, or small-scale societies; transegalitarian groups; complex communitiesall of these have been put forward as models and descriptors for situations in which social hierarchies, heterarchies, and complexity are present but not institutionalized or codified at a scale associated with states (Crumley 1995; Arnold 1996; Ames 2008; Porter 2013). In particular, emphasis on what societies do, rather than on what they are called, is useful in discussions of "tribal societies" or "village societies," which have existed from the Neolithic through the Modern period, often alongside more complex or urban political or settlement formations (Parkinson 2002; Bandy 2004; Bandy and Fox 2010). While the range of parlance presents certain challenges, such models can accommodate variability and dynamism in social organization. For example, the term "chiefdom" is often subject to critique because of the range of social formations it is deployed to describe (Yoffee 1993; Pauketat 2007). ${ }^{7}$ This might be seen rather as a strength, since it can highlight the variety of strategies and material manifestations of social groups exhibiting similar behaviors, as in Earle's (1997) comparative study of Denmark, Hawaii, and Peru, or in Beck's (2003) analysis of chiefdom variability in the Mississippian communities of the southeastern United States. While I do not adopt the term "chiefdom" here in any technical sense, I do draw on several studies of societies that have been defined as chiefdoms by other scholars, precisely because of this variability.

7. Debates about the appropriateness of the term "chiefdom" and its evolutionary implications are as old as Service's original formulation and do not need to be rehearsed here (Service 1962; Sahlins 1963; Fried 1967). For more recent literature on the subject, see Earle 1987, 1997; Kirch 2010, along with critiques by Yoffee 1993; Pauketat 2007. 
The archaeology of village societies is perhaps more salient for much of the early Greek landscape. Villages are typically defined as self-contained, relatively egalitarian social systems, which nevertheless have mechanisms for reaching beyond themselves. As is true of the neoevolutionary terms of band, tribe, and chiefdom, the definition is broad and can refer to a wide range of social formations (Fowles 2002; Gerstel 2020). In general, villages can be defined negatively as permanent communities that are not large enough to be urban. Territorial extents were probably not greater than they were required to be to meet local subsistence needs, and they were probably not clearly demarcated beyond the level of individual households, kin groups, and neighboring communities. In terms of sociopolitical complexity and regional integration, variation again is the rule, ranging from independent collections of a few households (the majority of cases) to more integrative modes of regional organization (in and around larger communities). Most significantly for this study, villages in the ancient Greek world are best understood through their rurality and their connections to the landscape and surrounding communities (Foxhall 2020).

Porter (2013) has proposed "complex communities" as a model for Early Iron Age societies in Jordan where complexity (1) is present, but variable across a culture area; (2) is dynamic; and (3) is not easily compared to contemporary or chronologically proximate political formations that are better known (e.g., Near Eastern states and empires). I have adopted this terminology throughout this book as a means of describing the range of societal formations witnessed in early Greece, which range across a sliding scale of being more or less hierarchical and politically complex. This terminology builds on the work of McGuire and Saitta (1996), who proposed "communal complexity" as a framework for understanding how communities oscillate between more hierarchical and more egalitarian. This terminology also acknowledges the capacity for entirely different modes of political organization to coexist simultaneously across the landscape. The appeal here is a flexible model for social complexity that can apply across a range of periods and social formations without prescribing certain sets of characteristics or teleological narratives.

Fowles (2002) has argued convincingly for a shift in comparative emphasis from types of entire societies to types of historical processes or societal trajectories. Trajectories similar to those witnessed in early Greek societies can be seen at different times in the fortress-building cultures of the Late Bronze Age Caucasus and the subsequent Urartian civilization (Smith 2003), in Mississippian chiefdoms (Blitz 2010), in maritime chiefdoms in Pacific and other contexts (Kirch 2010; Ling, Earle, and Kristiansen 2018), and in other modes of tribal organization (Parkinson 2002). Whether we call early Greek societies secondary states, chiefdoms, early complex polities, tribal societies, village societies, or complex communities is not especially important to the arguments in this book. Relative matters of scale, complexity, and development are important, however, and for this some 
use of analogy can be fruitful. Complex communities and village societies provide starting points, not so much as strict terminology but as a way of breaking free from restrictive narratives concerning states, palaces, or poleis. While critiques of this sort have a long history in other regions and are certainly part of scholarship on the Aegean, alternative models remain few. By thinking beyond state structures to landscape, interaction, and societal dynamics, there is an opportunity also for the Aegean to contribute to other world archaeologies, especially ones exhibiting nonlinear trajectories and a range of modes of social organization. 